Published in final edited form as:

J Am Chem Soc. 2016 February 17; 138(6): 1844-1847. doi:10.1021/jacs.5b13382.

\title{
Ligand induced conformational changes of a membrane transporter in E. coli cells observed with DEER/PELDOR
}

\author{
Benesh Joseph ${ }^{*}$, , Arthur Sikora§, and David S. Cafiso $\$,{ }^{*}$ \\ tInstitute of Physical and Theoretical Chemistry and Center for Biomolecular Magnetic \\ Resonance, University of Frankfurt, Max-von-Laue-Strasse 7, 60438 Frankfurt am Main, Germany \\ $\S$ Department of Chemistry and Center for Membrane Biology, University of Virginia, McCormick \\ Road, Charlottesville VA22904-4319, USA
}

\begin{abstract}
An unrealized goal in structural biology is the determination of structure and conformational change at high resolution for membrane proteins within the cellular environment. Pulsed electronelectron double resonance (PELDOR) is a well-established technique to follow conformational changes in purified membrane protein complexes. Here we demonstrate the first proof of concept for the use of PELDOR to observe conformational changes in a membrane protein in intact cells. We exploit the fact that outer membrane proteins usually lack reactive cysteines and the fact that paramagnetic spin labels entering the periplasm are selectively reduced to achieve specific labeling of the cobalamin transporter BtuB in Escherichia coli. We characterize conformational changes in the second extracellular loop of BtuB upon ligand binding and compare the PELDOR data with high-resolution crystal structures. Our approach avoids detergent extraction, purification and reconstitution usually required for these systems. With this approach structure, function, conformational changes and molecular interactions of outer membrane proteins can be studied at high resolution in the cellular environment.
\end{abstract}

\section{Graphical Abstract}

Corresponding Authors: joseph@epr.uni-frankfurt.de, cafiso@virginia.edu.

Notes

The authors declare no competing financial interests.

Supporting Information

Details on the whole cell preparation, spin-labeling, MTSL reduction kinetics, CW-EPR, PELDOR measurements and data analysis are presented. 


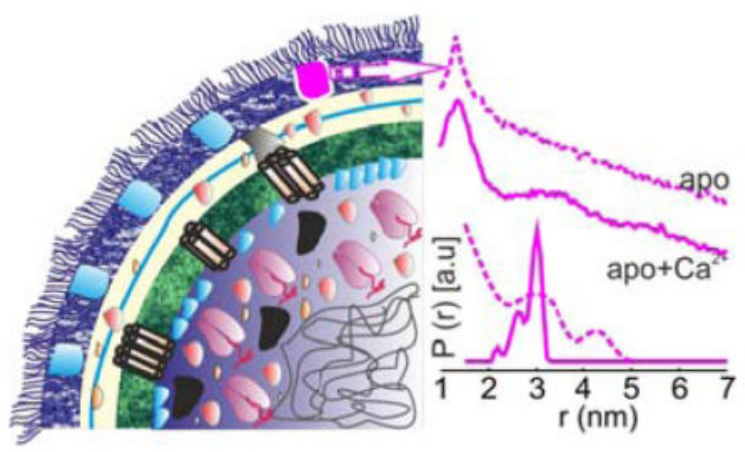

Determining biomolecular structures and their conformational changes at high resolution has primarily been achieved with X-ray crystallography, NMR spectroscopy and more recently with cryo-EM. In many cases these studies have provided detailed models for conformational transitions that drive function in many macromolecules including several membrane proteins. In almost all these cases, the structures have been obtained after isolating the target molecule from its native environment. This approach masks the effect of the cellular conditions such as molecular crowding, specific localization, interaction with other molecules/ions, $\mathrm{pH}$ or ionic gradients, the lipid environment and physiological responses. All these factors may critically influence the structure, function and dynamics of a biomolecule. For membrane proteins, there is increasing evidence on the vital role of the native lipid environment on protein folding, structure and activity. ${ }^{1,2}$ Thus, the next challenge for membrane protein structural biology is to obtain structural and dynamic information in the cellular environment.

Membrane proteins are often extracted and purified in detergent and are typically returned to a lipid environment by reconstituting the protein into a lipid bilayer consisting of native or non-native lipids. In this manner, conformational changes in several membrane proteins have been studied with EPR, ${ }^{3,4}$ FRET $^{5}$ and NMR. ${ }^{6}$ In-cell FRET usually employs fluorescent proteins as tags, and due to their large size these tags can provide only low resolution information. ${ }^{5}$ In-cell NMR experiments are limited due to low sensitivity and the requirement that the macromolecule be small and rapidly tumbling. ${ }^{7}$ The requirement for a well-ordered 2D crystal severely limits the use of diffraction techniques to study membrane proteins in native environments. Thus obtaining high resolution in-cell information for membrane proteins remains a challenge that necessitates new approaches.

Pulsed electron-electron double resonance (PELDOR or DEER $)^{8,9}$ has received a great deal of attention in structural biology, particularly for membrane proteins. The technique can resolve distance distributions between spin pairs with high precision in the range of 1.5 to $10.0 \mathrm{~nm} .{ }^{4,10,11}$ Combined with simulations and modeling, these distance distributions can validate existing structures and provide information on alternate structural states that have not been observed in crystals. ${ }^{12,13}$ PELDOR experiments are typically performed at low temperature, although under proper conditions they can be carried out in liquid solutions using spin labels having long phase memory time $\left(T_{m}\right) \cdot{ }^{14-16}$ Since proteins are usually diamagnetic, paramagnetic spin labels, most commonly the methan-ethiosulfonate spin label (MTSSL/MTSL), are introduced into a protein by covalent attachment to an engineered 
cysteine residue. ${ }^{17}$ Spin-labeling of a cysteine with MTSL generates the side chain named R1. Recently, Gd(III) spin labels have attracted attention for in-cell PELDOR because they are resistant to the reducing environment inside the cell. ${ }^{18,19}$ The use of PELDOR in-cell has been demonstrated using spin-labeled ubiquitin, which was introduced into oocytes or HeLa cells. ${ }^{19,20}$ Distance measurements have been reported on spin labeled colicin A added to $E$. coli. $^{21}$ However, the signal-to-noise ratio was poor and such exogenous introduction of a protein does not work for most of the membrane proteins. Recently, we used PELDOR to measure distances between the endogenous cobalamin transporter and its spin-labeled substrate in intact E. coli. ${ }^{22}$

Here we report ligand-induced conformational changes in the extracellular loops of the outer membrane cobalamin transporter BtuB in intact E. coli. To our knowledge, this is the first report of conformational changes in a membrane transporter at high resolution in the cellular environment. We show the potential of this approach to verify conformational changes observed in crystal structures and to obtain additional information in the cellular environment. Beta-barrel proteins are ubiquitous in gram-negative bacteria, chloroplasts and mitochondria and they perform vital physiological functions such as membrane biogenesis, substrate and protein translocation, motility, drug resistance, and signaling. BtuB is a 22stranded $\beta$-barrel protein that is filled with a 130 residue $\mathrm{N}$-terminal plug or hatch domain in the center. It is a member of the TonB-dependent transporter (TBDT) family, requiring a proton motive force (pmf) and the inner membrane ExbB-ExbD-TonB complex for cyanocobalamin $(\mathrm{CN}-\mathrm{Cbl})$ transport. We expressed BtuB in E. coli having two cysteines at the desired positions that were spin-labeled by adding MTSL to the cell suspension. Outer membrane $(\mathrm{OM})$ proteins in E. coli are often cysteine-free (cys-less) or have cysteines that are not reactive. Thus labeling of engineered solvent-accessible target sites could be achieved by adding MTSL to the cell exterior. ${ }^{22,23}$ However, it is not necessarily obvious why we should have achieved specific labeling.

The outer membrane is permeable to molecules below $600 \mathrm{Da}$, and the MTSL could easily reach periplasm and react with exposed cysteines of inner membrane proteins (Figure 1a). However, we have never observed an EPR signal following labeling of E. coli cells expressing 'cys-less' BtuB or other cysteine mutations located in periplasm. Attempts to label cysteines at the periplasmic interface with maleimido-proxyl also did not give a signal, ruling out any possible interference from the disulfide bond formation (Dsb) system. These observations suggest that the MTSL is reduced following entry into the periplasm. We demonstrated previously that a spin-labeled $\mathrm{CN}-\mathrm{Cbl}$, which binds tightly to $\mathrm{BtuB}$, is not reduced by cells ${ }^{22}$ confirming that the reduction must happen only after entry into the periplasm.

To further understand the reduction process, we added $150 \mu \mathrm{M}$ MTSL to a $30 \mathrm{~mL}$ suspension containing cells collected from a two liter overnight culture. Samples were collected at periodic intervals and MTSL concentration in the supernatants was monitored using room temperature continuous wave EPR spectroscopy (RT CW-EPR). As shown in Figure 2a, the spin concentration in the supernatant quickly decreased reaching less than 10 $\mu \mathrm{M}$ in 45 minutes. Similar results have been observed for the reduction of Tempone and spin-labeled gentamycin by Pseudomonas aeruginosa and E. coli cells ${ }^{24}$ and for the TPOA 
spin label (2,2,5,5-tetramethyl-pyrroline-1-oxyl-3-carboxylic acid amide) in Xenopus laevis oocytes. ${ }^{25}$ Greater stability has been reported for another modified five-membered nitroxide spin-label in E. coli. ${ }^{26}$ It is possible that the rather high concentration of the spin-label (1020 fold) compared to what we used and other experimental conditions might have contributed to the greater stability. The apparent MTSL reduction was much faster for 188C-399C mutant. At present we do not understand the reasons for this observation and additional work is needed to determine whether the presence of unfolded precursors with reactive cysteines in the periplasm might have accelerated MTSL reduction.

As we reported earlier, no signal could be detected for the WT cell pellet ${ }^{22}$ or for cysteine mutations located in periplasm (8C, 9C, Figure S2), whereas single or double cysteine mutants located on the extracellular loops or the exposed surface of the hatch domain yielded signal from bound MTSL (Figure 2c-e). We conclude that upon entry into periplasm, MTSL may stay free, react with accessible cysteines or even cross the inner membranes. In any case it is quickly reduced. As suggested previously, ${ }^{24}$ interaction with the electron transport chain in the inner membrane might be a reason for the rapid reduction. Whatever the exact mechanism of MTSL reduction is, this process eliminated signals from unwanted sites, which is very critical for the PELDOR experiment.

Despite the availability of several crystal structures, the mechanism for substrate transport remains unclear for TBDTs. ${ }^{27} \mathrm{In}$ all the structures, the $\mathrm{N}$-terminal hatch domain occludes the barrel leaving no space for substrate movement. It has been suggested that the $\mathrm{N}$ terminal domain may remain within the barrel and rearrange ${ }^{28}$ or exit the barrel partially ${ }^{29}$ or completely ${ }^{30}$ during translocation. It is possible that a native cellular environment is necessary for the $\mathrm{N}$-terminal domain to achieve a translocation-competent conformation. We attempted to spin label positions $66 \mathrm{C}, 74 \mathrm{C}$ and $90 \mathrm{C}$ located on the N-terminal domain in BtuB (Figure 2b). We could label $74 \mathrm{C}$ and $90 \mathrm{C}$ whereas labeling failed for $66 \mathrm{C}$ probably because it is sterically restricted as observed in the crystal structures (Figure S2c). Interestingly, both 74R1 and 90R1 revealed spectra in the rigid limit (correlation time $\left(\tau_{c}\right)>$ $100 \mathrm{~ns}$, Figure S3) suggesting a static conformation for the N-terminal domain in the cellular environment. In future, it will be interesting to explore how this rigid conformation is modulated by the presence of ligands, pmf or TonB in the cellular environment.

To investigate the possibility of observing conformational changes in intact $E$. coli cells, we spin labeled 188C-399C located on the 2nd extra cellular loop (connecting $\beta$-strands 3 and 4 ) and 7th loop (connecting $\beta$-strands 13 and 14), respectively. The 2nd loop shows very large conformational changes in the crystal structures in response to ligand binding. ${ }^{31}$ It is not resolved in the BtuB-apo crystal structure, whereas it is completely ordered in both BtuB-Ca ${ }^{2+}$ and BtuB-Ca ${ }^{2+}-\mathrm{CN}-\mathrm{Cbl}$ structures (Figure $2 \mathrm{~b}$ ). BtuB binds two $\mathrm{Ca}^{2+}$ ions with high affinity through an aspartate cage consisting of several residues from loops 2 and 3. Loop 7 carrying position 399 is well ordered even in the absence of the ligands and has a very similar conformation in all the three crystal structures (Figure $2 \mathrm{~b}$ ). Thus changes in the 188R1-399R1 distance are expected to result from motion of the 2nd loop.

Unlike for the positions located on the hatch domain, RT CW-EPR spectroscopy revealed a rather mobile spectrum for 188R1-399R1 in agreement with their location on the loop sites 
(Figure 2e). For the 188R1 single mutant, we could achieve up to $30 \mu \mathrm{M}$ spin concentration using a $2 \times 10^{11}$ cells $/ \mathrm{mL}$ suspension. ${ }^{22}$ However, for the 188R1-399R1 double mutant we could obtain only $30 \mu \mathrm{M}$ spin (instead of the expected $60 \mu \mathrm{M}$ ) at the same cell density. The modulation depth $(\lambda)$ of the PELDOR traces for the in-cell samples presented below is in the 6-8 \% range (Figure 3). This is about $25 \%$ of the maximum $\lambda$ achievable with our Q-band instrument for a sample with $100 \%$ spin-labeling efficiency. Thus we obtained only 50-60\% spin-labeling efficiency for the 188C-399C double mutant in E. coli cells. There could be several reasons for this low labeling efficiency in whole cells. The surrounding LPS molecules may interfere with MTSL accessibility (Figure 1a), and since the reduction appears to be very fast (Figure 2a), some reduced MTSL might have diffused back from periplasm and reacted with $188 \mathrm{C}$ and $399 \mathrm{C}$. In spite of the low spin concentration and $\lambda$, we could achieve high quality PELDOR data in intact E. coli cells with 36-48 hrs of accumulation. BtuB single cysteine mutants do not show any distances in the measurable range confirming that BtuB exists as a monomer in the cellular environment. ${ }^{22}$ The PELDOR data were analyzed using Deer-Analysis software ${ }^{32}$ employing Tikhonov regularization with L-curve criterion and the error of the distance distribution was estimated by systematically varying the intermolecular background function (Figure S4-5).

In the apo-state (no $\mathrm{Ca}^{2+}$ or $\mathrm{CN}-\mathrm{Cbl}$ ) in E. coli cells, 188R1-399R1 cells showed a broad interspin distance distribution with a mean distance at $2.66 \pm 0.9 \mathrm{~nm}$ (Figure $3 \mathrm{~d}$, red). It is likely that the dynamic nature of the 2nd loop contributes to the broad conformational distribution and explains why this loop is not resolved in the BtuB-apo crystal structure (Figure 2b). Thus in the absence of $\mathrm{Ca}^{2+}$, the 2nd loop occupies a large conformational landscape in $E$. coli cells and might completely occlude the binding pocket in some states. Interestingly, addition of $\mathrm{Ca}^{2+}$ ions to the cells populated a distinct conformation with a mean distance at $2.82 \pm 0.30 \mathrm{~nm}$ (Figure 3e, red), in agreement with the appearance of the 2nd loop in BtuB-Ca ${ }^{2+}$ structure. ${ }^{31}$ Simulation of this spin pair in the context of this crystal structure (1NQG) using $\mathrm{MMM}^{33}$ gave a predicted distribution that was broader with a mean distance at $2.61 \pm 0.39 \mathrm{~nm}$ (Figure 3e, cyan). Further addition of $\mathrm{CN}-\mathrm{Cbl}$ to form the ternary complex shifted the main peak giving a mean distance at $3.12 \pm 0.33 \mathrm{~nm}$. Simulation on the corresponding crystal structure $(1 \mathrm{NQH})$ predicted a broader distribution with a mean distance at $2.73 \pm 0.37 \mathrm{~nm}$ (Figure 3f, cyan). Despite the differences between the experimental results and the simulations, the PELDOR data validated the $\mathrm{Ca}^{2+}$-induced ordering of the 2nd loop observed in the crystal structures in the cellular environment.

To further investigate the role of the cellular environment, we isolated outer membranes $(\mathrm{OM})$ containing BtuB using an established protocol ${ }^{34}$ (see methods). PELDOR with the OM revealed (Figure 3d-e, black) some differences compared to the whole cell samples in the apo- and $\mathrm{Ca}^{2+}$-bound states. In the apo-sate, the overall distance distributions are similar, however in whole cells there is a higher population of shorter distances (mean distance of $2.6 \pm 0.89 \mathrm{~nm}$ as compared to $3.16 \pm 0.84 \mathrm{~nm}$ in $\mathrm{OM}$ ). In the $\mathrm{Ca}^{2+}$-bound state, the $2 \mathrm{nd}$ loop again becomes ordered (mean distances at $2.72 \pm 0.31 \mathrm{~nm}$ ) as observed in whole cells and in the crystal structure. The shape of the distance distribution in OM appears different when compared to whole cells. In presence of both $\mathrm{Ca}^{2+}$ and $\mathrm{CN}-\mathrm{Cbl}$, the distances obtained in OM $(2.98 \pm 0.18 \mathrm{~nm})$ were virtually identical to the whole cell samples (Figure 3f). Notably in $\mathrm{OM}$, the presence of both $\mathrm{Ca}^{2+}$ and $\mathrm{CN}-\mathrm{Cbl}$ induced a narrower distance distribution as 
compared to $\mathrm{Ca}^{2+}$ alone. In summary, there exist small differences in the distance distribution between whole cell and OM environments; however the overall responses of the loops to ligand(s) binding are very similar. Similar conformational changes were observed previously in POPC vesicles, ${ }^{27}$ however, the quality of this earlier data was not comparable to that obtained here and a quantitative comparison with the previous result was not made. The differences observed between the simulations and the experiment could be explained by the exclusion of some of the rotamers (populated in the crystals) in the native membrane environment (Figure 3e-f, cyan vs. red and black). Such selective rotamer exclusion might result from additional steric interference around the spin labels perhaps due to interactions with LPS, proteins or due to other components present in the cellular environment. The limited accuracy in the prediction of the rotamers and the interspin distances $( \pm 3 \AA)^{33}$ may also have contributed to the differences.

The data presented here demonstrate that conformational changes in a membrane protein can be observed using PELDOR in an isolated native membrane. The OM PELDOR data suggest that isolation of the outer membrane using the standard procedure does not severely alter the behavior of BtuB. Moreover, when compared to whole cells, the OM samples are very stable and may be concentrated allowing higher quality PELDOR data and shorter acquisition times (Figure 3). In addition, these OM preparations are leaky and provide access to both membrane surfaces, a feature that will be useful for studying interactions between OM proteins and other molecular partners located in the periplasm or inner membrane (with BtuF or TonB for example) or to investigate interaction and folding of proteins into native outer membranes. In summary, we demonstrated the measurement of conformational changes in a membrane protein within the native cellular environment of intact $E$. coli cells for the first time. Our results reveal a very dynamic conformation of the 2nd loop in the apo-state that is not resolved in the crystal structure. Further, we validated the conformational changes of this loop upon ligand binding observed in BtuB crystal structures in the cellular environment. Two important features of the $E$. coli cells make this observation possible; the lack of native reactive cysteines in OM proteins and the selective reduction of MTSL that enters the periplasm.

With its higher sensitivity and ability to examine structures of any molecular weight, PELDOR is ideally suited to obtain distance constraints in the cellular environment. In principle any solvent exposed sites other than those located in the periplasm can be spinlabeled. Thus, residues on the extra-cellular loops or the $\mathrm{N}$-terminal domain which are involved in substrate recognition and translocation in numerous $\beta$-barrel proteins could be spin-labeled and studied in intact cells. Additionally, these proteins could be genetically modified to incorporate paramagnetic metal binding tags or amino acids in the periplasm. The general applicability of the method would be extended with further improvement in sensitivity. The signal can be increased with optimization of the labeling efficiency and by using stronger and tunable promoters to increase expression levels. Addition of $\mathrm{d}_{8}$-glycerol can improve $T_{m}$ in both OM and whole cell samples, ${ }^{22}$ and it might be possible to increase the sensitivity by growing cells in a deuterated media. Normally, BtuB is expressed at less than $10^{3}$ copies/cell and the overexpression used here increases the expression up to $10^{5}$ copies/cell. ${ }^{22}$ Several OM proteins are expressed at more than $10^{5}$ copies per cell, thus it should be possible to study these proteins at physiological concentrations in the cellular 
environment. Combined with the recent developments in pulsed EPR instrumentation and pulse sequences, ${ }^{35-37}$ it will be possible to perform PELDOR at even lower expression levels.

\section{Supplementary Material}

Refer to Web version on PubMed Central for supplementary material.

\section{Acknowledgments}

This work was financially supported by the Deutsche For-schungsgemeinschaft (SFB 807) and Marie-Curie GO-IN Fellowship (B.J.) and NIGMS grant GM035215 (D.S.C). B.J would like to thank Prof. T.F. Prisner for the critical discussions and support and Prof. Enrico Schleiff for providing the protein expression facilities.

\section{References}

1. Laganowsky A, Reading E, Allison TM, Ulmschneider MB, Degiacomi MT, Baldwin AJ, Robinson CV. Nature. 2014; 510:172. [PubMed: 24899312]

2. Dowhan W, Bogdanov M. Biochem Soc Trans. 2011; 39:767. [PubMed: 21599647]

3. Klare, JP.; Steinhoff, H-J. In Methods in Enzymology. Peter, ZQ.; Kurt, W., editors. Vol. 564. Academic Press; Cambridge, MA: 2015. p. 315

4. Jeschke G. Ann Rev Phys Chem. 2012; 63:419. [PubMed: 22404592]

5. Piehler J. Cur Opin Struct Biol. 2014; 24:54.

6. Hong M, Zhang Y, Hu F. Annu Rev Phys Chem. 2012; 63:1. [PubMed: 22136620]

7. Hansel R, Luh LM, Corbeski I, Trantirek L, Dotsch V. Angew Chem. 2014; 53:10300. [PubMed: 25070284]

8. Milov AD, Ponomarev AB, Tsvetkov YD. Chem Phys Lett. 1984; 110:67.

9. Pannier M, Veit S, Godt A, Jeschke G, Spiess HW. J Magn Reson. 2000; 142:331. [PubMed: 10648151]

10. Schiemann O, Prisner TF. Quart Rev Biophys. 2007; 40:1.

11. Ward R, Bowman A, Sozudogru E, El-Mkami H, Owen-Hughes T, Norman DG. J Magn Reson. 2010; 207:164. [PubMed: 20805036]

12. Claxton, DP.; Kazmier, K.; Mishra, S.; Mchaourab, HS. Methods in Enzymology. Peter, ZQ.; Kurt, W., editors. Vol. 564. Academic Press; Cambridge, MA: 2015. p. 349

13. Joseph B, Korkhov VM, Yulikov M, Jeschke G, Bordignon E. J Biol Chem. 2014; 289:3176. [PubMed: 24362024]

14. Yang Z, Liu Y, Borbat P, Zweier JL, Freed JH, Hubbell WL. J Am Chem Soc. 2012; 134:9950. [PubMed: 22676043]

15. Meyer V, Swanson MA, Clouston LJ, Boratynski PJ, Stein RA, Mchaourab HS, Rajca A, Eaton SS, Eaton GR. Biophys J. 2015; 108:1213. [PubMed: 25762332]

16. Shevelev GY, Krumkacheva OA, Lomzov AA, Kuzhelev AA, Rogozhnikova OY, Trukhin DV, Troitskaya TI, Tormyshev VM, Fedin MV, Pyshnyi DV, Bagryanskaya EG. J Am Chem Soc. 2014; 136:9874. [PubMed: 24963806]

17. Hubbell WL, Altenbach C. Cur Opin Struct Biol. 1994; 4:566.

18. Qi M, Gross A, Jeschke G, Godt A, Drescher M. J Am Chem Soc. 2014; 136:15366. [PubMed: 25325832]

19. Martorana A, Bellapadrona G, Feintuch A, Di Gregorio E, Aime S, Goldfarb D. J Am Chem Soc. 2014; 136:13458. [PubMed: 25163412]

20. Igarashi R, Sakai T, Hara H, Tenno T, Tanaka T, Tochio H, Shirakawa M. J Am Chem Soc. 2010; 132:8228. [PubMed: 20513154]

21. Dunkel S, Pulagam LP, Steinhoff HJ, Klare JP. PCCP. 2015; 17:4875. [PubMed: 25613578] 
22. Joseph B, Sikora A, Bordignon E, Jeschke G, Cafiso DS, Prisner TF. Angew Chem. 2015; 54:6196. [PubMed: 25826642]

23. Jiang X, Payne MA, Cao Z, Foster SB, Feix JB, Newton SM, Klebba PE. Science. 1997; 276:1261. [PubMed: 9157886]

24. Rapoport N, Smirnov AI, Pitt WG, Timoshin EA. Arch Biochem Biophys. 1999; 362:233. [PubMed: 9989932]

25. Krstic I, Hansel R, Romainczyk O, Engels JW, Dotsch V, Prisner TF. Angew Chem. 2011; 50:5070. [PubMed: 21506223]

26. Schmidt MJ, Borbas J, Drescher M, Summerer D. J Am Chem Soc. 2014; 136:1238. [PubMed: 24428347]

27. Cafiso DS. Acc Chem Res. 2014; 47:3102. [PubMed: 25152957]

28. Chakraborty R, Storey E, van der Helm D. Biometals. 2007; 20:263. [PubMed: 17186377]

29. Devanathan S, Postle K. Mol Microbiol. 2007; 65:441. [PubMed: 17578453]

30. Ma L, Kaserer W, Annamalai R, Scott DC, Jin B, Jiang X, Xiao Q, Maymani H, Massis LM, Ferreira LC, Newton SM, Klebba PE. J Biol Chem. 2007; 282:397. [PubMed: 17056600]

31. Chimento DP, Mohanty AK, Kadner RJ, Wiener MC. Nat Struct Biol. 2003; 10:394. [PubMed: 12652322]

32. Jeschke G, Chechik V, Ionita P, Godt A, Zimmermann H, Banham J, Timmel CR, Hilger D, Jung H. Appl Magn Reson. 2006; 30:473.

33. Polyhach Y, Bordignon E, Jeschke G. PCCP. 2011; 13:2356. [PubMed: 21116569]

34. Filip C, Fletcher G, Wulff JL, Earhart CF. J Bacteriol. 1973; 115:717. [PubMed: 4580564]

35. Polyhach Y, Bordignon E, Tschaggelar R, Gandra S, Godt A, Jeschke G. PCCP. 2012; 14:10762. [PubMed: 22751953]

36. Spindler PE, Waclawska I, Endeward B, Plackmeyer J, Ziegler C, Prisner TF. J Phys Chem Lett. 2015; 6:4331. [PubMed: 26538047]

37. Borbat PP, Georgieva ER, Freed JH. J Phys Chem Lett. 2013; 4:170. [PubMed: 23301118] 
a

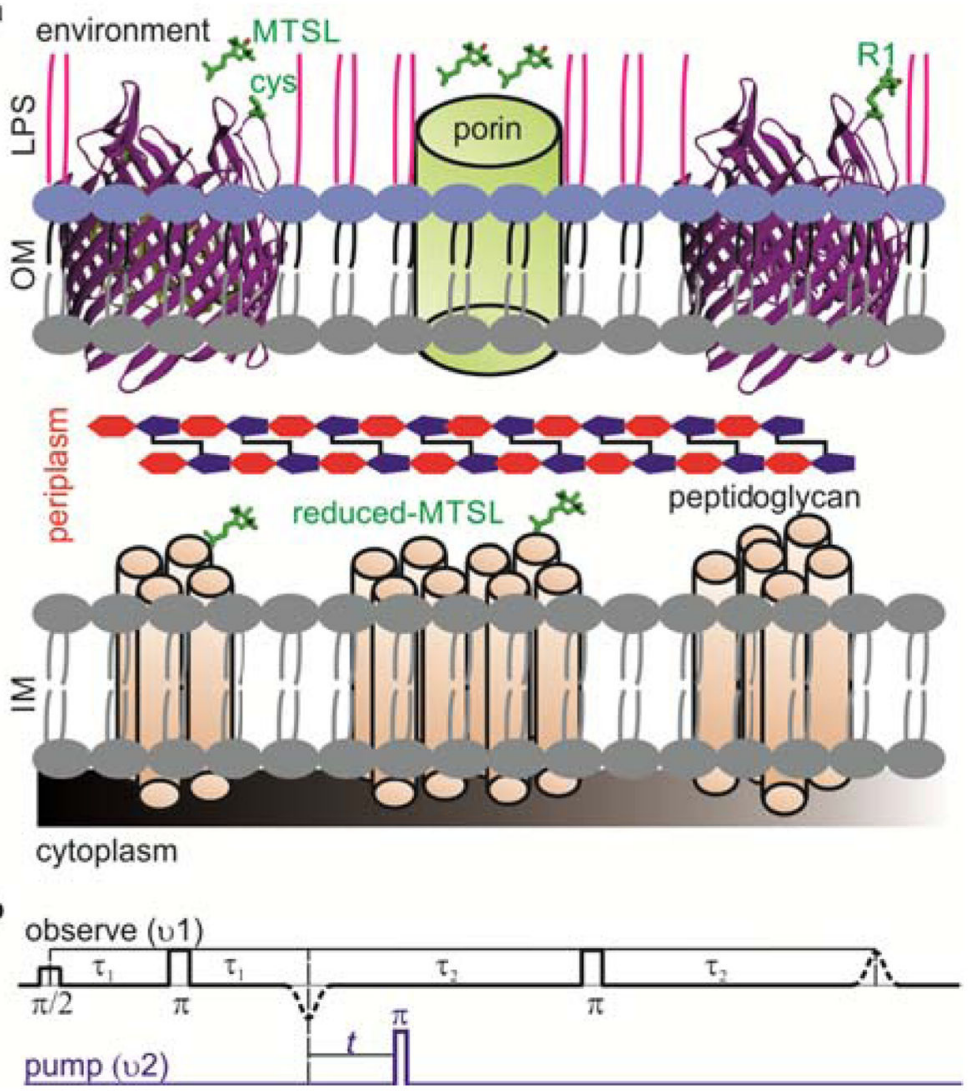

Figure 1.

(a) Structure of the the Gram-negative bacterial cell wall. The outer membrane (OM) is assymetric consisting of an inner phospholopid layer and an outer lipopolysacharide (LPS) layer. The inner membrane (IM) consists of a phosopholipid bilayer containing numerouns a-helical proteins. Exposed cysteines on $\beta$-barrel proteins can be labeled by additon of MTSL from outside. Those MTSL molecules which enter periplasm through the porins are reduced. (b) The PELDOR (4-pulse DEER) ${ }^{9}$ pulse sequence consists of a refocused echo at the observer frequency (black) and its intensity modulation is monitored as a function of the timing of an inversion pulse at the pump frequency (blue). 

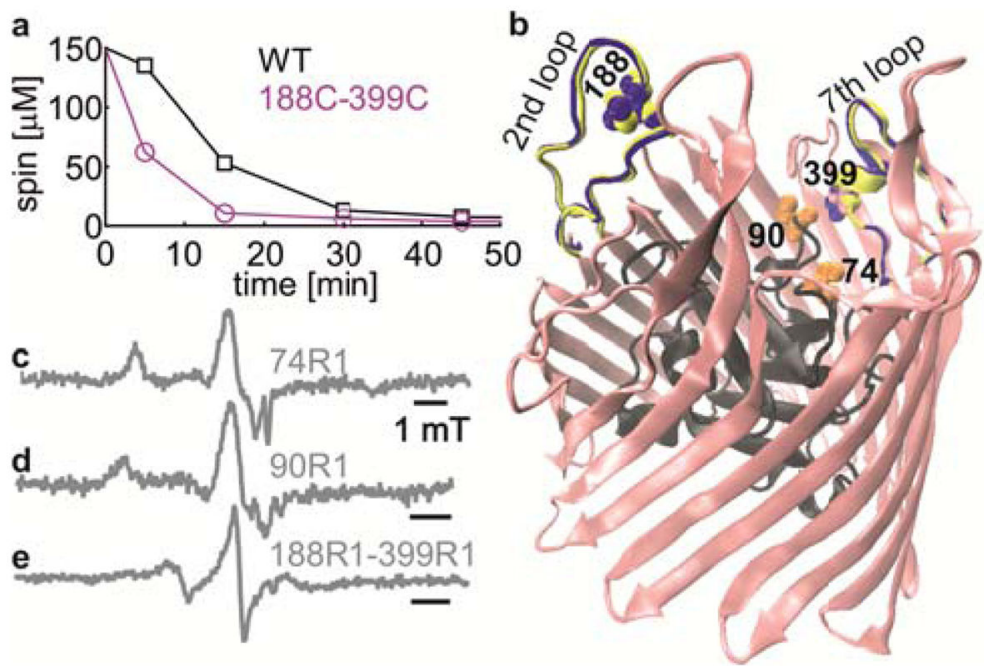

Figure 2.

(a) Reduction of MTSL by E. coli cells expressing WT or 188C-399C BtuB. A 10-15\% error is estimated for the spin concentration calculated using RT CW W-EPR. (b) apo-BtuB structure (1NQE) with the plug domain in black. Spin-labeled positions are highlighted in $\mathrm{CPK}$ representation. The $2 \mathrm{nd}$ extra cellular loop conformation as observed in the $\mathrm{BtuB}-\mathrm{Ca}^{2+}$ (blue, $1 \mathrm{NQG}$ ) and BtuB-Ca ${ }^{2+}-\mathrm{CN}-\mathrm{Cbl}$ (yellow, $1 \mathrm{NQH}$ ) is overlaid. (c, d, e) RT CW-EPR spectra measured in live E. coli $\left(2 \times 10^{9}\right.$ cells $)$ as s indicated. 
a
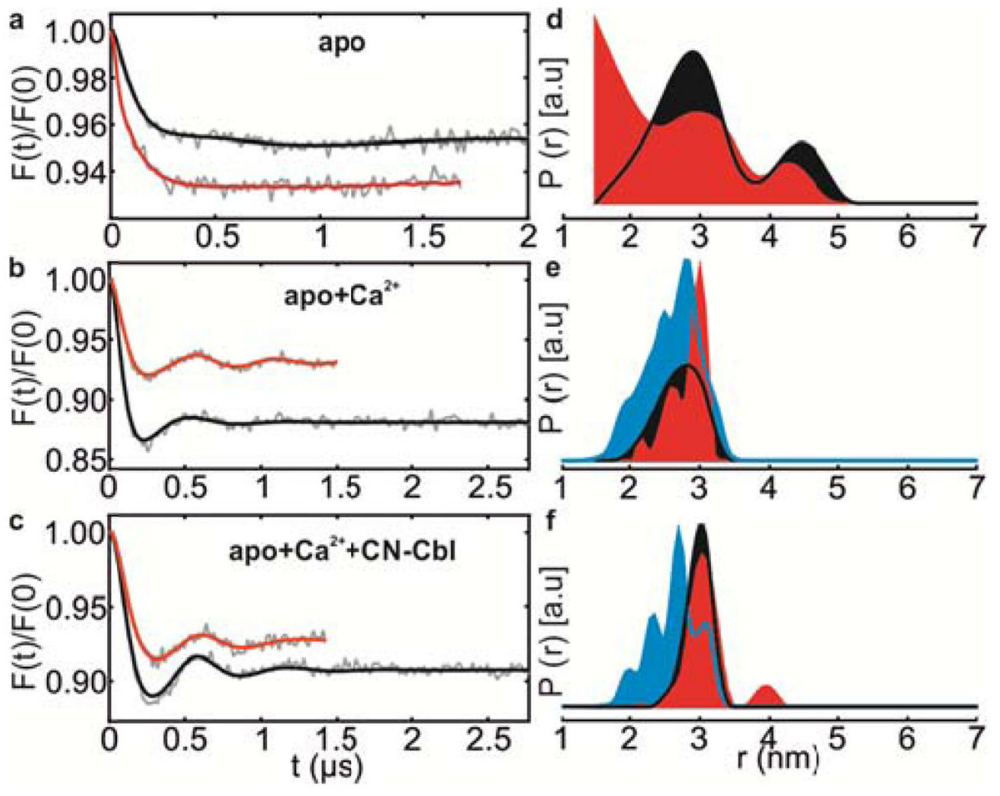

Figure 3.

(a-c) Background corrected Q-band PELDOR data at $50 \mathrm{~K}$ for 188R1-399R1 mutant in $E$. coli cells (red) or OM (black) in different functional states. (d-f) Area normalized distance distributions and the corresponding simulations (cyan) normalized to the maximum of the experimental data. 